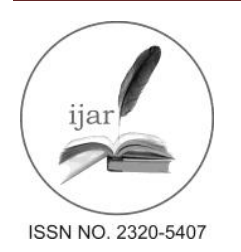

Journal homepage:http://www.journalijar.com
Journal DOI:10.21474/IJAR01

RESEARCH ARTICLE
INTERNATIONAL JOURNAL

OF ADVANCED RESEARCH

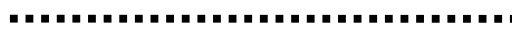

\title{
PREVALENCE OF HAIR LOSS AND STRESS AS THE CAUSE; A CROSS-SECTIONAL STUDY.
}

\author{
Soorih Shaikh, Sarwan Shaikh, Sarees Shaikh, Aijaz Ali Shaikh and Syed Ghazanfar Saleem.
}

The Indus Hospital Karachi.

\section{Manuscript Info}

Manuscript History:

Received: 15 May 2016

Final Accepted: 14 June 2016

Published Online: July 2016

Key words:

Hair loss; Stress; Perceived Stress

Scale; Hair Pull Test.

*Corresponding Author

Soorih Shaikh.

\begin{abstract}
Background: Limited research has been undertaken on the Prevalence of hair lossand its relationship to stress in Pakistan. This cross-sectional study attempts at knowing the prevalence of hair loss among general population and to determine the relationship between stress and hair loss.

Methods: We conducted a cross-sectional study. A randomized sample of both male and female of all ages from different communities were offered to participate in the study. All the participants were assessed for level of stress using 14-item Sheldon Cohen 'Perceived Stress Scale (PSS-14)' and tested by 'Hair Pull Test' for hair loss. PSS scores for low stress were set ranging from 0-18, scores ranging from 19-36 wereconsidered moderate stress and scores ranging from 37-56 corresponded to high stress.

Results: A total of 456 individuals were invited and 424 [including both male (236) and female (188)] of them participated in the study, with a response rate of $92.9 \%$.

As per study criteria, the results of stress test showed that $67(15.8 \%)$ individuals had low stress, $279(65.8 \%)$ had moderate stress while 78 (18.3\%) had high stress. A total of $42(9.9 \%)$ individuals fulfilled the criteria of excessive hair loss (more prevalent among males in general; 30 out of 236 males (12.7\%):(6.3\%) 12 out of 188 females). In Males, hair loss was seen in $7.5 \%$ individuals with low stress, $9.8 \%$ individuals with moderate stress and $32 \%$ individuals with high stress. While in Females, hair loss was seen in $3.7 \%$ individuals with low stress, $5.9 \%$ individuals with moderate stress and $9.0 \%$ individuals with high stress).
\end{abstract}

Conclusion:Hair loss is more prevalent in the male population (males show 2 times more hair loss than females) and there is a strong relationship between stress and hair loss.

\section{Introduction:}

Hair is one of the characteristic of mammals and has an undeniable importance in personality. People have between 100,000 and 150,000 hairs on their head. The number of strands normally lost in a day varies, but on average is 50100. This usually doesn't cause noticeable hair loss because new hair is growing in at the same time. If this growthloss cycle is disrupted, then hair loss (alopecia or baldness) is seen. Hair loss can be related to several factors, including Family history (Androgenic alopecia or Patterned hair loss), Hormonal changes (Thyroid dysfunction, pregnancy/ childbirth, menopause), Alopecia areata ('Spot Baldness'- autoimmune process that results in hair follicles' dormancy and loss), scalp infection (ringworm etc.), skin disorders affecting scalp (lupus etc.), trichotillomania ('Hair pulling disorder'- irresistible urge to pull out one's hair, whether it's from the scalp, the eyebrows or other areas of the body), Radiation/Chemo therapy, excessive hair-styling (traction etc.), and Stress (telogen effluvium)etc.

Any kind of physical trauma such as surgery, a car accident, child birth or a severe illness, even the flu, can cause 
temporary hair loss. This can trigger a type of hair loss called 'telogen effluvium'. Hair has a programmed life cycle: a growth phase (anagen), resting phase (catagen) and shedding phase (telogen). Soon a person goes through stressful event, hairs can go into the resting phase. Hair loss often becomes noticeable two-to-six months after the trauma.

We know that stress can, either directly or indirectly, affect functioning of almost every organ of the body; but stress is the primary cause of hair loss or only a factor leading to various other factors such as loss of sleep, loss of appetite and increase in stress hormones responsible for hair loss is still debated.

Our aim was to study this relationship between stress and hair loss and the prevalence of Hair loss in the general population.

\section{Methods and Materials}

We conducted a cross-sectional study. A randomized sample was taken from different places including schools, hospitals, private and government sector offices, gymnasium and relaxing parks, irrespective of age and gender. A total of 424 people participated out of 456 invited for the study. All the participants were assessed for their level of stress using 14-item Sheldon Cohen 'Perceived Stress Scale (PSS-14)' and were asked not to wash their hair for one day. On the following day, all the participants were subjected to 'Hair Pull Test' to assess degree of hair loss.

The PSS-14 is a self-reported measure which assesses the degree to which the respondent has perceived situations in his/her life within the past month as stressful. Individuals rate items on a 5-point scale, ranging from 0 as "never" to 4 as "very often". Scores range from 0-56, with higher scores indicating greater perceived stress. PSS scores for low stress were set ranging from 0-18, scores ranging from 19-36 were considered moderate stress and scores ranging from 37-56 corresponded to high stress.

Hair loss is assessed through various techniques, such as, Hair pull test, Hair pluck test, Daily hair counts, Wash test, Trichoscopy and Scalp biopsy etc. Among all, Hair Pull Test (HPT) is the simplest test to assess severity of diffuse scalp hair loss. About 50-60 hairs are hold in between thumb, index and middle fingers and a gentle but firm traction is exerted, on three different areas of the scalp (top/vertex, occiput and frontal area). 5 or less hair extracted in HPT is normal, while 6 or more hair extracted shows positive test and excessive hair loss.

\section{Data analysis}

SPSS version 2.0 was used foranalysis. Subjects with $\geq 6$ hairs on HPT were considered to have excessive hair loss. Statistical significance between the two was studied using Chi-square test. Significance level was set at $\mathrm{p}<0.05$.

Table 1a: Stress vs Hair loss relation (Males \& Females Combined)

\begin{tabular}{|l|l|l|l|l|}
\hline & LowStress & Moderate Stress & HighStress & Total \\
\hline Hair Loss Present & 4 & 23 & 15 & $\mathbf{4 2}$ \\
\hline Normal & 63 & 256 & 63 & $\mathbf{3 8 2}$ \\
\hline Total & $\mathbf{6 7}$ & $\mathbf{2 7 9}$ & $\mathbf{7 8}$ & $\mathbf{4 2 4}$ \\
\hline
\end{tabular}

Table 1b: Stress vs Hair loss relation (Males)

\begin{tabular}{|l|l|l|l|l|}
\hline & LowStress & Moderate Stress & HighStress & Total \\
\hline Hair Loss Present & 3 & 16 & 11 & $\mathbf{3 0}$ \\
\hline Normal & 37 & 146 & 23 & $\mathbf{2 0 6}$ \\
\hline Total & $\mathbf{4 0}$ & $\mathbf{1 6 2}$ & $\mathbf{3 4}$ & $\mathbf{2 3 6}$ \\
\hline
\end{tabular}

Table 1c: Stress vs Hair loss relation (Females).

\begin{tabular}{|l|l|l|l|l|}
\hline & Low Stress & Moderate Stress & High Stress & Total \\
\hline Hair Loss Present & 1 & 7 & 4 & $\mathbf{1 2}$ \\
\hline Normal & 26 & 110 & 40 & $\mathbf{1 7 6}$ \\
\hline Total & $\mathbf{2 7}$ & $\mathbf{1 1 7}$ & $\mathbf{4 4}$ & $\mathbf{1 8 8}$ \\
\hline
\end{tabular}




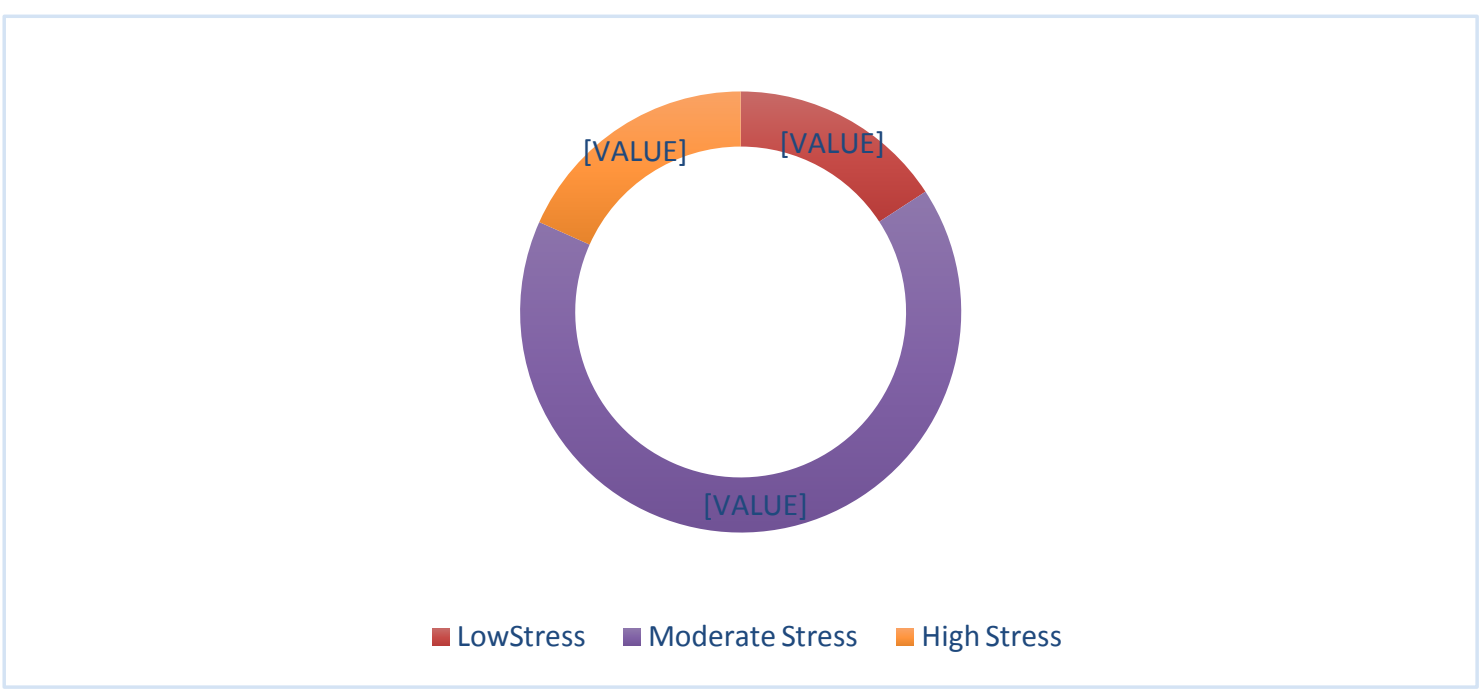

Fig 1: Stress Grading in General population (Males \& Females Combined)

Most of the general population lies in Moderate to high grade Stress.

Low Stress: (67/424)

Moderate Stress: (279/424)

High Stress: (78/424)

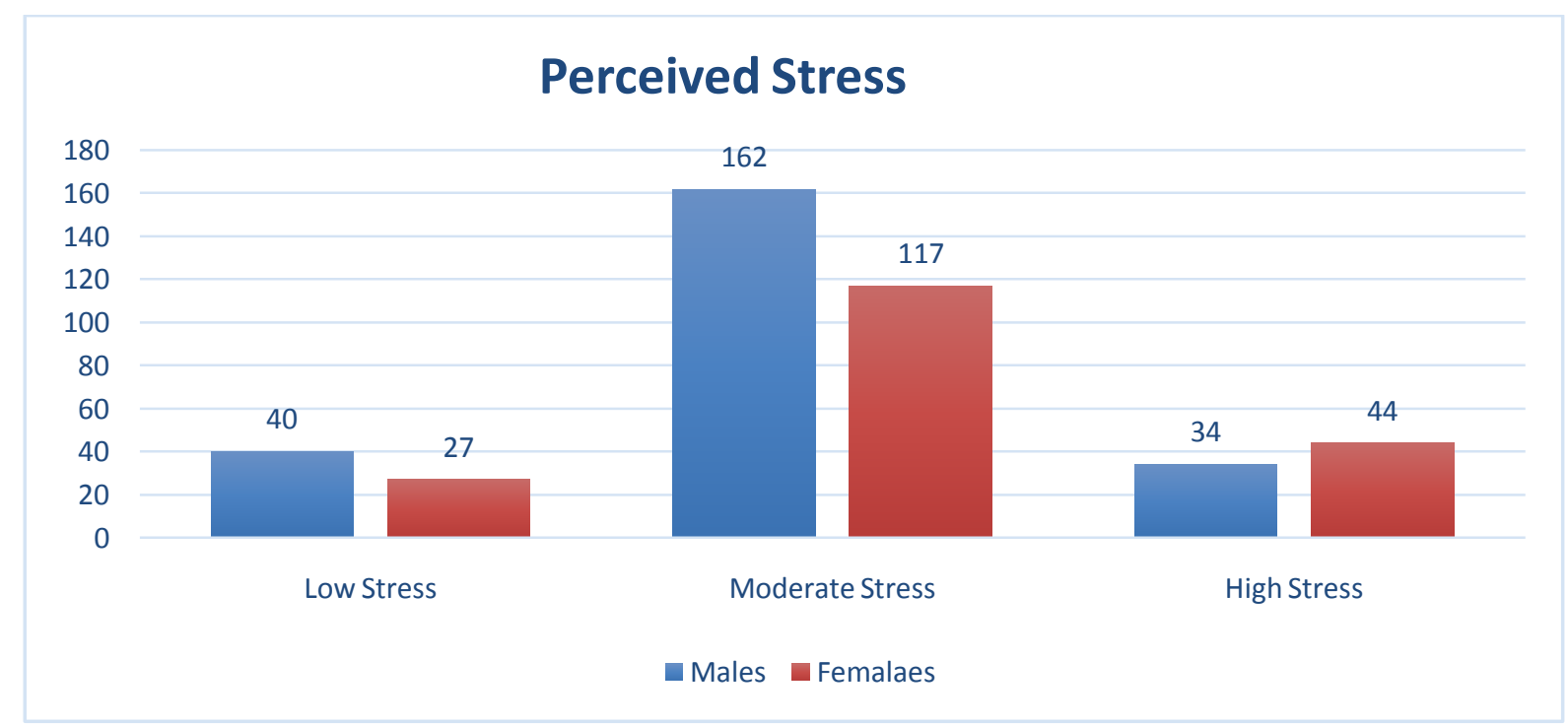

Fig 2: Perceived Stress prevalence; distributive chart in Males \& Females.

Low Stress: 40 males \& 27 females

Moderate Stress: 162 males \& 117 females

High Stress: 34 males \& 44 females

Males perceive more stress in the selected sample population. 


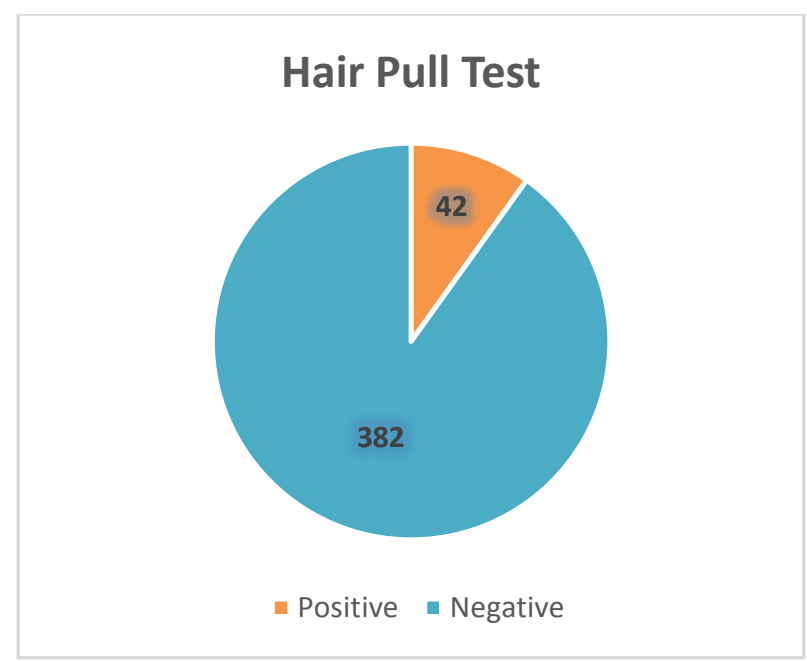

Fig 3: Hair Pull Test in general population.

Among 424 individuals participating in the study, 42 (9.9\%) show positive Hair Pull test result while 382 (90.1\%) show negative result.

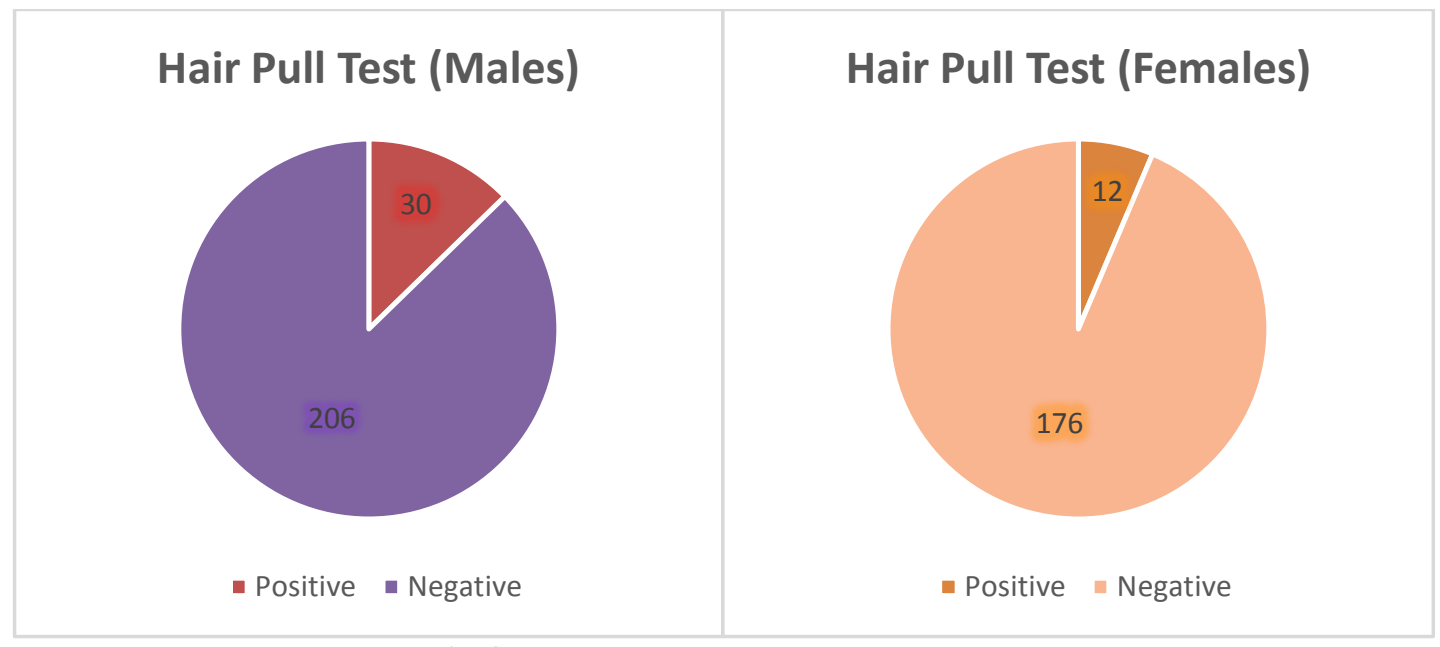

Fig 4: Hair Pull Test in Males Vs Females.

In male population of 236, 30 (12.7\%) individuals show positive Hair Pull Test result while 206 (87.3\%) show negative result;(Prevalence rate of $12.7 \%)$.

In female population of 188, only 12 (6.3\%) individuals show positive Hair Pull Test result while 176 (93.7\%) show negative result;(Prevalence rate of 6.3\%). 


\section{(Positive Hair Pull Test)}

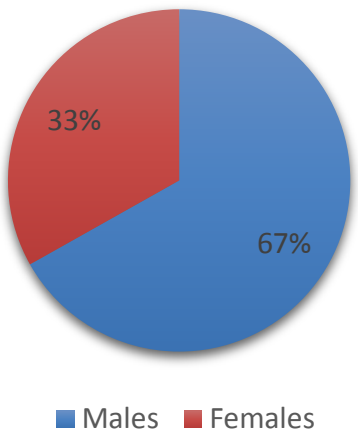

Fig 5: Male vs Female prevalence rate of Hair Loss (Positive Hair Pull Test)

Comparing male to female prevalence of hair loss $(12.7 \%$ vs $6.3 \%$, respectively); we find that males show 2 times more hair loss, irrespective of the cause.

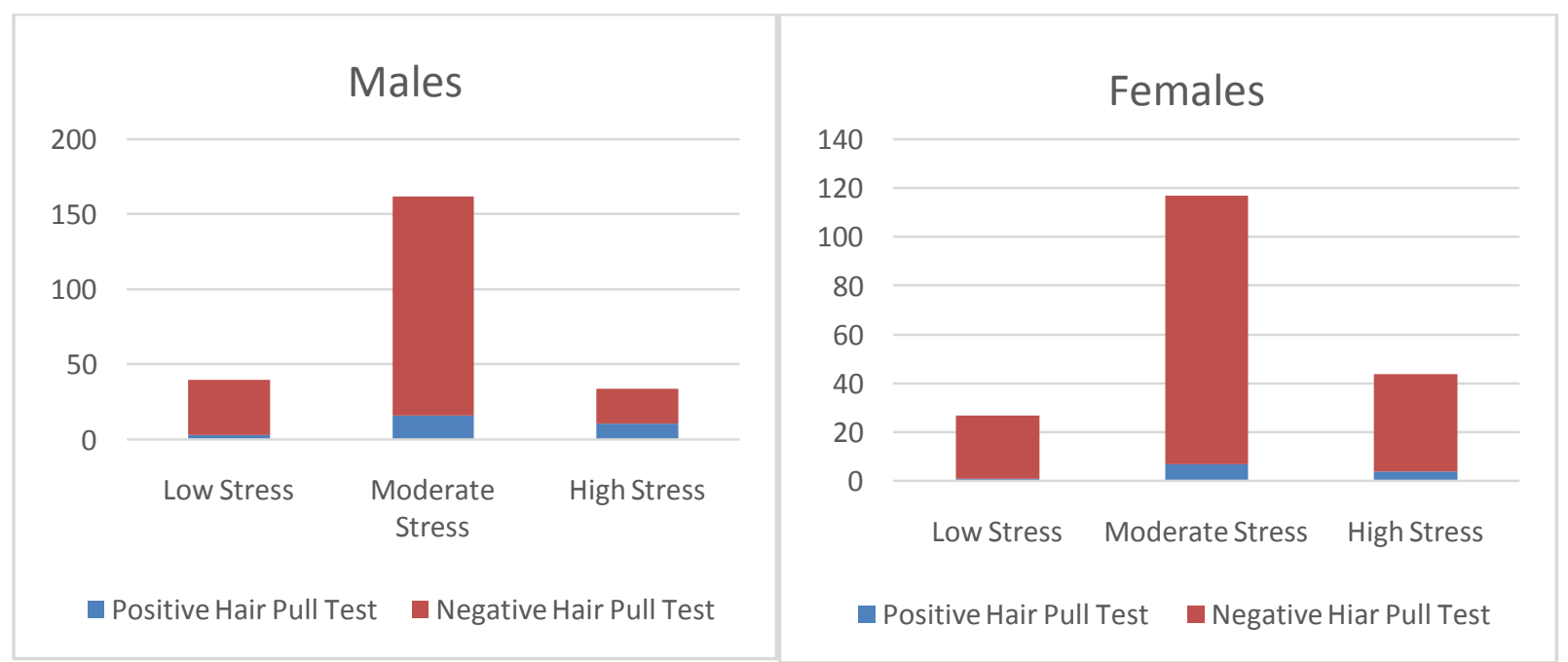

Fig 6: Stress vs Hair Loss (Positive Hair Pull) relation in Males and Females.

Comparatively, male population shows more hair loss (Positive Hair Pull Test) for any stress grade.

(In Males, hair loss was seen in $7.5 \%$ individuals with low stress, $9.8 \%$ individuals with moderate stress and $32 \%$ individuals with high stress. While in Females, hair loss was seen in $3.7 \%$ individuals with low stress, $5.9 \%$ individuals with moderate stress and $9.0 \%$ individuals with high stress).

\section{Results}

A total of 456 individuals were invited and 424 of them [including both male (236) and female (188)] participated in the study, a response rate of $92.9 \%$.

The results of stress test showed that 67 (15.8\%) individuals had low stress (40 males:27 females), $279(65.8 \%)$ had moderate stress (162 males:117 females) while 78 (18.3\%) had high stress (34 males:44 females).

A total of $42(9.9 \%)$ individuals fulfilled the criteria of excessive hair loss (more prevalent among males in general; 30 out of 236 males (12.7\%):(6.3\%) 12 out of 188 females).

Among those with hair loss (30 males and 12 females), in males 3 had low stress $(3 / 40=7.5 \%)$, 16 had moderate stress $(16 / 162=9.8 \%)$, and 11 had high stress $(11 / 34=32 \%)$ and in females 1 had low stress $(1 / 27=3.7 \%), 7$ had moderate stress $(7 / 117=5.9 \%), 4$ had high stress $(4 / 44=9.0 \%)$. 
A total of 10 males ( 2 with moderate $\& 8$ with high stress) and 3 females ( 1 with moderate $\& 2$ with high stress) with excessive hair loss had no explainable cause of hair loss, leaving only stress as the probable cause.

Using Chi-square $\left(\chi^{2}\right)$ test, significant difference was observed in frequencies of hair loss on the level of stress in males $(\mathrm{p}=0.018)$ and in females $(\mathrm{p}=0.032)$;

This implies that hair loss is more prevalent among the male population compared to the female population and stress has more effect on the hair loss in male population.

\section{Discussion}

Limited research has been undertaken on the Prevalence of hair lossand its relationship with stress in Pakistan. In this study, we have highlighted the prevalence and relationship of Hair loss and stress in general population. We used the Hair Pull Test to determine Hair loss and found the prevalence of excessive Hair loss in 42 (9.9\%) individuals from the general population (both males and females); selected from different ages, communities, places (offices, schools, hospitals etc.). We also used 'Sheldon Cohen-Perceived Stress Scale' to determine level of stress and found a high prevalence of stress in our study population, majority having moderate to high stress; out of 424 participating individuals, $279(65.8 \%)$ had moderate while $78(18.3 \%)$ had high stress.

Hair loss was seen in significant number of participants, more commonly in males, but almost all of them had explainable cause for hair loss, except for some male and a few female patients who for their age and history had more loss of hairs on their head, diffusely. These individuals also corresponded to high stress on PSS. It is plausible that stress related hair loss may be more common in male population due to increase in totalconcentration of steroid hormones, especially in stressful situations.

In a study conducted on female medical students to look for prevalence of stress and its relation to hair fall the selected population did not experience significant hair fall, hence, the relationship between stress and hair fall could not be made. In comparison, we chose a larger and diversified sample and hence got different and statistically significant results. We found that higher the stress is in any individual more is the hair loss.

Many studies have been conducted to state the relationship between stress and hair fall. Some have focused on stress causing hair fall, while others have looked for hair fall causing stress. One such study that focuses on hair fall causing stress was conducted in a public dermatological clinic, showing that out of 157 women interviewed, 54\% reported hair loss and 29\% reported at least two major symptoms of depression [14]. They concluded that complaints of hair loss were common and were associated with depression among adult female outpatients [14].

Although, our study results show therelation between hair loss and stress, but, since our study was focused only at knowing the relation between stress and hair loss, not the definitive reason why stress causes hair loss, we suggest that further studies should be conducted on specifically stressed populations to establish stronger relationship and to know the pathophysiology behind stress causing hair loss.

\section{Conclusion}

Hair loss is more prevalent in the male population (males show 2 times more hair loss than females) and there is a strong relationship between stress and hair loss. Males generally show more hair loss as compared to females because of steroid hormones (Testosterone). It is plausible that the release of higher amounts of steroid hormones in stress might be the cause of excessive hair loss in stressed populations. 


\section{References:-}

1. Zhang X, Caulloo S, Zhao Y, Zhang B, Cai Z, Yang J. Female pattern hair loss: clinico-laboratory findings and trichoscopy depending on disease severity. Int JTrichology 2012; 4:23-8.

2. Shrivastava SB. Diffuse hair loss in an adult female: approach to diagnosis and management. Indian JDermatolVenereolLeprol 2009; 75:20-7; quiz 27-8.

3. York J, Nicholson T, Minors P, Duncan DF. Stressful life events and loss of hair among adult women, a casecontrol study. Psychol Rep 1998; 82:1044-6.

4. Rushton DH, Dover R, Norris MJ, Gilkes JJ. Iron and hair loss in women; what is deficiency? This is the real question! J Am AcadDermatol 2007; 56:518-9; author reply 519.

5. Nnoruka EN. Hair loss: is there a relationship with hair care practices in Nigeria.Int J Dermatol 2005; 44

6. McMullen R, Jachowicz J. Image analysis of hair treated with styling products. J CosmetSci 2004; 55:128-9.

7. Gummer CL. Hair shaft effects from cosmetics and styling. ExpDermatol 1999; 8:317.

8. O'Rourke M, Hammond S, O'Flynn S, Boylan G. The Medical Student Stress Profile: a tool for stress audit in medical training. Med Educ 2010; 44:1027-37.

9. Lee BS, Chan JY, Monselise A, McElwee K, Shapiro J. Assessment of hair density and caliber in Caucasian and Asian female subjects with female pattern hair loss by using the Folliscope. J Am AcadDermatol 2012; 66:166-7.

10. Olszewska M, Warszawik O, Rakowska A, Slowinska M, Rudnicka L. Methods of hair loss evaluation in patients with endocrine disorders. Endokrynol Pol 2010; 61:406-11.

11. Sinclair R, Patel M, Dawson TL Jr, Yazdabadi A, Yip L, Perez A, Rufaut NW. Hair loss in women: medical and cosmetic approaches to increase scalp hair fullness. Br J Dermatol 2011; 165 Suppl 3:12-8.

12. Shapiro J, Wiseman M, Lui H. Practical management of hair loss. Can Fam Physician 2000; 46:1469-77.

13. Cohen S, Kamarck T, Mermelstein R. A global measure of perceived stress. J Health SocBehav 1983; 24:38596.

14. Schmitt JV, Ribeiro CF, Souza FH, Siqueira EB, Bebber FR. Hair loss perception and symptoms of depression in female outpatients attending a general dermatology clinic. An Bras Dermatol 2012; 87:412-7. 